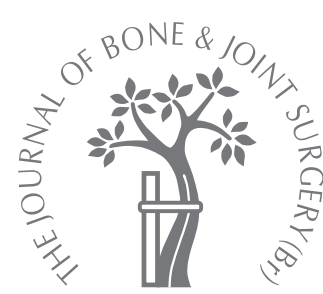

S.-T. Jeong, H.-R. Song, S. M. Keny, S. S. Telang, S.-W. Suh, S.-J. Hong

From Korea University Medical Center, Guro Hospital, Seoul, Korea

S.-T. Jeong, MD, PhD Associate Professor Department of Orthopaedics Gyeong-Sang National University, Jinju, Korea.

H.-R. Song, MD, PhD, Professor

S. M. Keny, MS, Fellow S. S. Telang, MS, DNB, Fellow

- S.-W. Suh, MD, PhD, Associate Professor Department of Orthopaedics, Rare Disease Institute = S.-J. Hong, MD, PhD, Associate Professor Department of Radiology Korea University, Guro Hospital, \# 80, Guro-Dong, Guro-Gu, Seoul 152-703, Korea.

Correspondence should be sent to $\mathrm{Dr}$ H.-R. Song; e-mail: songhae@korea.ac.kr

(C2006 British Editorial Society of Bone and Joint Surgery doi:10.1302/0301-620X.88B9. $17758 \$ 2.00$

$J$ Bone Joint Surg $[\mathrm{Br}]$ 2006;88-B:1192-6.

Received 10 February 2006; Accepted after revision 17 May 2006

\title{
MRI study of the lumbar spine in achondroplasia
}

\author{
A MORPHOMETRIC ANALYSIS FOR THE EVALUATION OF \\ STENOSIS OF THE CANAL
}

We carried out an MRI study of the lumbar spine in 15 patients with achondroplasia to evaluate the degree of stenosis of the canal. They were divided into asymptomatic and symptomatic groups. We measured the sagittal canal diameter, the sagittal cord diameter, the interpedicular distance at the mid-pedicle level and the cross-sectional area of the canal and spinal cord at mid-body and mid-disc levels.

The MRI findings showed that in achondroplasia there was a significant difference between the groups in the cross-sectional area of the body canal at the upper lumbar levels. Patients with a narrower canal are more likely to develop symptoms of spinal stenosis than others.

Neurological problems are present in 35\% to $47 \%$ of patients with achondroplasia. ${ }^{1,2}$ There may be a delay in mental and motor development, hypotonia, feeding and sleep disorders, and compressive spinal syndromes in children, but adults usually present with symptoms of spinal stenosis. ${ }^{1,2}$ These are associated with considerable debility and reduction in the quality of life.

The cause of spinal canal stenosis in achondroplasia is a disorder of enchondral ossification which results in early fusion of the pedicles to the vertebral bodies at the neurocentral synchrondosis. ${ }^{1-6}$ The cross-sectional area of the spinal canal is consequently narrowed by the shortened pedicles and decreased interpedicular distance, which leaves a reduced space available for the neural elements.

A number of studies have described the morphometry of the bony and the neural elements of the normal spine. However, to our knowledge, such a study is lacking for patients with achondroplasia. ${ }^{7,8}$ The usual spinal dimensions in such patients make it difficult for the spinal surgeon to assess the degree of lumbar spinal stenosis and to plan treatment.

We have carried out a morphometric MRI analysis of the achondroplastic lumbar spine in an attempt to define its usual dimensions and thereby to provide a reference for future studies. We examined five dimensions of the lumbar spine to determine whether they could help to distinguish between symptomatic and asymptomatic patients.

\section{Patients and Methods}

Fifteen patients with achondroplasia were divided into two groups, based on whether they were symptomatic or not. The symptomatic group consisted of eight patients (three men, five women) who had symptoms and signs suggestive of stenosis of the lumbar canal. The asymptomatic group consisted of seven patients (two men, five women) without symptoms or signs suggestive of stenosis. The mean age of the symptomatic group was 32 years (21 to 47$)$ and that of the asymptomatic group was 26 years (17 to 43 ). Patients who had symptoms of nerve-root compression without symptoms of claudication and those with a severe high lumbar kyphosis were excluded.

All the patients completed an extensive questionnaire which included questions on the symptoms of low back pain, their duration, and aggravating and relieving factors. A history of lower-limb paraesthesiae, motor weakness, use of analgesics, and bladder symptoms and their duration were also recorded.

The patients then underwent a detailed clinical and neurological examination by the senior surgeon (HRS) which included a sciatic and femoral stretch test and an examination of the muscle power in the lower limbs, the feeling in each lumbar and sacral dermatome and the presence or absence of reflexes.

MR imaging was performed using a 1.5 Tesla T scanner (Sonata; Siemens Medical System, Erlanger, Germany). Slices $3 \mathrm{~mm}$ thick were taken and measurements were made using a digital software program (Piview Star, 


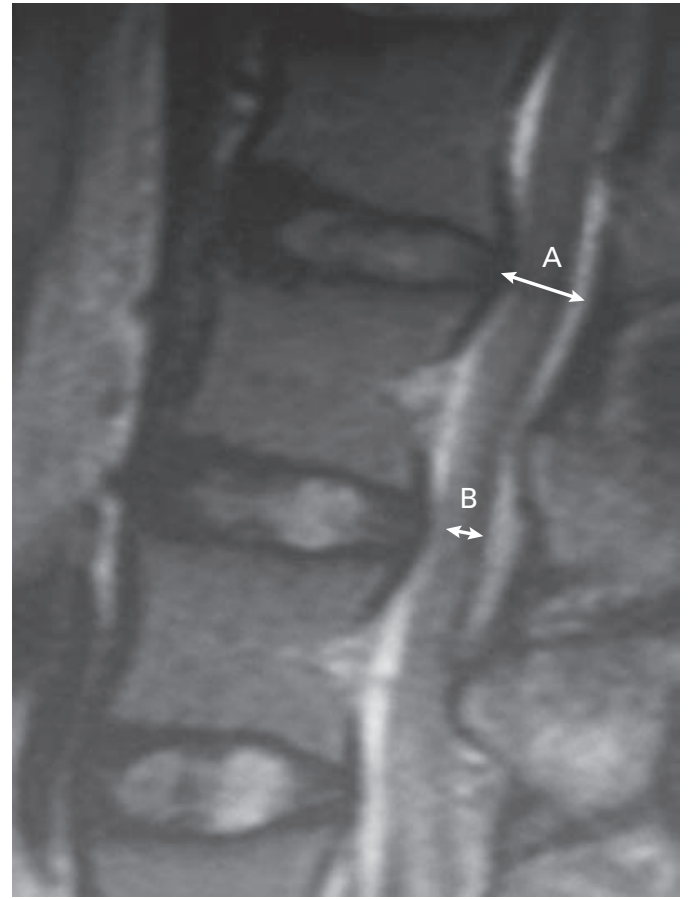

Fig. 1

Mid-sagittal T2-weighted MR image of the lumbar spine in an asymptomatic patient, showing A) the sagittal spinal canal diameter and B) the sagittal diameter of the cauda equina.

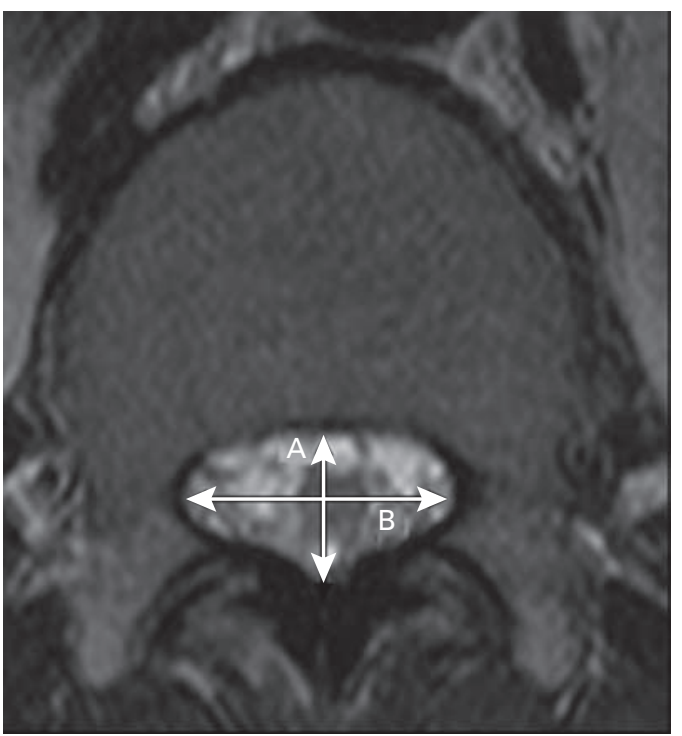

Fig. 2

MR image demonstrating the anteroposterior (A) and transverse dimensions (B).

Star Pacs Infinitt, Seoul, Korea). T1- and T2-weighted sagittal and axial cuts were obtained. Measurements were carried out by an experienced radiologist (SJH) specialising in MRI of the spine. On the T2-weighted sagittal images, the diameters of the spinal canal and the cauda equina at the mid-vertebral and the mid-disc levels from L1 to S1 were measured (Fig. 1). The anterior border of the spinal canal was taken to be the anterior border of the cerebrospinal fluid column on MRI (best visualised on a T2-weighted axial image), while the posterior border of the spinal canal was taken as the posterior margin of the cerebrospinal column. The column was used as a reference for measuring the diameter of the canal, since it could be clearly delineated from the surrounding structures on the T2-weighted image. The epidural space is very narrow in achondroplasia and it can therefore be very difficult to differentiate between the bony canal and thecal sac. The interpedicular distance was measured on the T2-weighted image at the mid-pedicle level from L1 to L5.

The cross-sectional area of the spinal canal and the cauda equina were also measured at the mid-point of each intervertebral level and at the mid-body level in the midaxial MR image site. Measurements of the anteroposterior (A) and transverse dimensions (B) in millimetres (Fig. 2) were made and the calculation $(\mathrm{A} \times \mathrm{B})$ performed using the method described by Yukawa et al. ${ }^{3}$

Statistical analysis. The diameter and the cross-sectional area of the spinal canal and the dural sac at the mid-body and mid-disc levels between the asymptomatic and symptomatic patients were compared using the Mann-Whitney Utest. A p value $<0.05$ was considered to be significant. All statistical analyses were performed using SPSS (version 12.0) software (SPSS Inc., Chicago, Illinois).

\section{Results}

The findings are summarised in Table I.

Sagittal diameter and interpedicular distance. The mean sagittal diameter of the spinal canal and the thecal sac and the mean interpedicular distance at the mid-body and the middisc levels, were not significantly different (L1 to L5 p = 0.613 to $\mathrm{p}=0.152$ ) between symptomatic and asymptomatic patients except at the mid-body level at L1 ( $\mathrm{p}=$ 0.009).

Distribution of stenosis. In the symptomatic group, the cross-sectional area of the canal was only compromised at the intervertebral disc levels. Each patient had a combination of stenosis of the central canal and of the lateral recess. No patient had significant dural compression at the level of the vertebral body as a result of stenosis of the canal. The most common levels affected were L1/2 and L3/4.

Cross-sectional area of the spinal canal. The cross-sectional area of the spinal canal was significantly different between symptomatic and asymptomatic patients at both the midbody and the mid-disc levels of L1 and L3 and at the midbody level of L2 (mid-body $\mathrm{p}=0.006$; mid-disc $\mathrm{p}=0.035$ ). Cross-sectional area of the cauda equina. The cross-sectional area at the mid-body level showed a progressive decrease from L1 to L5 in both asymptomatic and symptomatic patients. At the mid-disc level, it decreased progressively from L1 to L3 and then increased at L5 in both groups. 
Table I. Comparative analysis of the mean (median), SD of the diameter of the lumbar canal and thecal sac, interpedicular distance and the crosssectional area of the spinal canal and the cauda equina, at the mid-body and mid-disc levels in the asymptomatic and symptomatic groups of patients

\begin{tabular}{|c|c|c|c|c|c|}
\hline & L1 & L2 & L3 & L4 & L5 \\
\hline \multicolumn{6}{|c|}{ Canal diameter (mm) } \\
\hline \multicolumn{6}{|l|}{ Mid-body } \\
\hline Asymptomatic & 12.8 (13.0) SD 1.3 & $12.3(12.5)$ SD 1.3 & $11.6(11.1)$ SD 2.2 & $12.1(11.5)$ SD 4.1 & $11.8(12.0)$ SD 3.1 \\
\hline Symptomatic & $10.7(11.1)$ SD 1.5 & $11.6(11.9)$ SD 1.8 & $11.7(11.7)$ SD 1.2 & $11.3(11.5)$ SD 1.0 & $11.6(11.6)$ SD 1.2 \\
\hline $\mathrm{p}$ value & 0.009 & 0.418 & 0.487 & 0.728 & 0.886 \\
\hline \multicolumn{6}{|l|}{ Mid-disc } \\
\hline Asymptomatic & $9.9(10.9)$ SD 2.1 & $10.7(10.7)$ SD 1.8 & $10.5(10.8)$ SD 1.8$)$ & $10.2(10.4)$ SD 3.5 & $11.3(10.2)$ SD 4.5 \\
\hline Symptomatic & 9.1 (8.9) SD 1.7 & $9.0(9.6)$ SD 2.0 & $10.1(10.4)$ SD 1.8 & $9.8(10.3)$ SD 2.1 & $11.1(11.0)$ SD 2.6 \\
\hline $\mathrm{p}$ value & 0.355 & 0.165 & 0.728 & 0.699 & 0.881 \\
\hline \multicolumn{6}{|c|}{ Thecal diameter (mm) } \\
\hline \multicolumn{6}{|l|}{ Mid-body } \\
\hline Asymptomatic & $7.3(7.4)$ SD 1.0 & $7.2(7.1)$ SD 1.9 & $6.7(6.6)$ SD 1.7 & $6.5(6.0)$ SD 1.7 & $8.1(6.0)$ SD 4.6 \\
\hline Symptomatic & $6.7(6.7)$ SD 2.0 & 7.5 (7.7) SD 2.0 & 7.5 (7.6) SD 1.4 & $7.4(7.2)$ SD 1.7 & 7.7 (7.7) SD 2.0 \\
\hline$p$ value & 0.297 & 0.817 & 0.355 & 0.223 & 0.917 \\
\hline \multicolumn{6}{|l|}{ Mid-disc } \\
\hline Asymptomatic & $6.2(6.4)$ SD 1.1 & $7.2(6.7)$ SD 1.7 & $6.7(5.7)$ SD 2.6 & $7.0(5.9)$ SD 2.6 & $7.51^{*}$ \\
\hline Symptomatic & $6.1(6.5)$ SD 1.6 & $7.2(7.2)$ SD 1.3 & $6.8(6.9)$ SD 1.5 & 7.0 (7.7) SD 2.1 & $7.2(6.2)$ SD 1.5 \\
\hline $\mathrm{p}$ value & 0.908 & 0.817 & 0.685 & 0.770 & 0.770 \\
\hline \multicolumn{6}{|c|}{ Interpedicular distance (mm) } \\
\hline \multicolumn{6}{|c|}{ Mid-pedicle } \\
\hline Asymptomatic & $22.4(22.2)$ SD 1.23 & 21.9 (22.3) SD 1.85 & $20.9(20.2)$ SD 2.75 & $21.2(21.4)$ SD 3.25 & $22.0(21.4)$ SD 3.94 \\
\hline Symptomatic & $22.9(23.1)$ SD 1.58 & $20.3(20.2)$ SD 1.10 & $19.8(20.0)$ SD 0.69 & 18.6 (18.3) SD 1.51 & $18.6(18.9)$ SD 2.72 \\
\hline $\mathrm{p}$ value & 0.613 & 0.189 & 0.867 & 0.189 & 0.152 \\
\hline \multicolumn{6}{|l|}{ Canal area $\left(\mathrm{mm}^{2}\right)$} \\
\hline \multicolumn{6}{|l|}{ Mid-body } \\
\hline Asymptomatic & 241 (228) SD 29 & 197 (188) SD 50 & 189 (184) SD 50 & $143(121)$ SD 50 & 117 (92) SD 66 \\
\hline Symptomatic & 175 (174) SD 32 & $155(120)$ SD 58 & $128(122)$ SD 46 & 128 (133) SD 40 & $90 \quad$ (88) SD 17 \\
\hline$p$ value & 0.006 & 0.110 & 0.048 & 0.565 & 0.473 \\
\hline \multicolumn{6}{|l|}{ Mid-disc } \\
\hline Asymptomatic & 135 (133) sd 30 & 117 (115) SD 41 & 134 (116) SD 66 & 115 (102) SD 32 & 119 (109) SD 57 \\
\hline Symptomatic & 92 (101) sd 37 & 72 (67) SD 15 & 77 (72) SD 25 & 89 (72) SD 27 & $90 \quad(94)$ SD 24 \\
\hline $\mathrm{p}$ value & 0.035 & 0.018 & 0.035 & 0.142 & 0.224 \\
\hline \multicolumn{6}{|c|}{ Cauda equna area $\left(\mathrm{mm}^{2}\right)$} \\
\hline \multicolumn{6}{|c|}{ Mid-body } \\
\hline Asymptomatic & 175 (175) SD 12 & 142 (133) SD 29 & $126(121)$ SD 23 & 109 (101) SD 26 & 107 (86) SD 38 \\
\hline Symptomatic & $152(144)$ SD 40 & 127 (114) SD 39 & 112 (110) SD 29 & 112 (107) SD 23 & 85 (91) SD 24 \\
\hline $\mathrm{p}$ value & 0.048 & 0.277 & 0.225 & 0.749 & 0.568 \\
\hline \multicolumn{6}{|l|}{ Mid-disc } \\
\hline Asymptomatic & 93 (85) SD 16 & 79 (72) SD 19 & 83 (87) SD 23 & 84 (79) SD 19 & $110(100)$ SD 51 \\
\hline Symptomatic & 65 (65) SD 10 & 61 (61) SD 13 & 60 (58) SD 17 & 72 (77) SD 15 & 75 (74) SD 23 \\
\hline $\mathrm{p}$ value & 0.002 & 0.142 & 0.055 & 0.210 & 0.074 \\
\hline
\end{tabular}

However, a significant difference between symptomatic and asymptomatic patients was seen at both the mid-body and the mid-disc levels only at the L1 level (mid-body $\mathrm{p}=0.048$; mid-disc $\mathrm{p}=0.002$ ).

\section{Discussion}

Achondroplasia is a relatively common genetic disorder with an incidence of 1 per 13500 live births. It is associated with various abnormalities of the musculoskeletal system including developmental stenosis of the cervical and lumbar canals because of a defect in vertebral development, ${ }^{4,5}$ with premature fusion of the posterior elements. The normal spinal canal, as measured by its interpedicular distance, widens from L1 to L5, but in patients with achondroplasia this distance progressively decreases.
If a developmentally narrow canal is the cause of the stenosis and its associated symptoms, the cauda equina should be compressed at the level of the vertebral body. In our study, all the patients in the symptomatic group, however, had stenosis at the level of the intervertebral disc, suggesting that the stenosis was degenerative (Fig. 3). Hence, a developmentally narrow canal and early or accelerated degenerative change seem to be the most important factors for the development of symptomatic stenosis of the lumbar canal in achondroplasia.

In our symptomatic patients neural compression was because of protrusion of the intervertebral disc and hypertrophy of the ligamentum flavum. Each patient had a combination of stenosis of the central canal and the lateral recess. None had significant compression of the dural sac at 


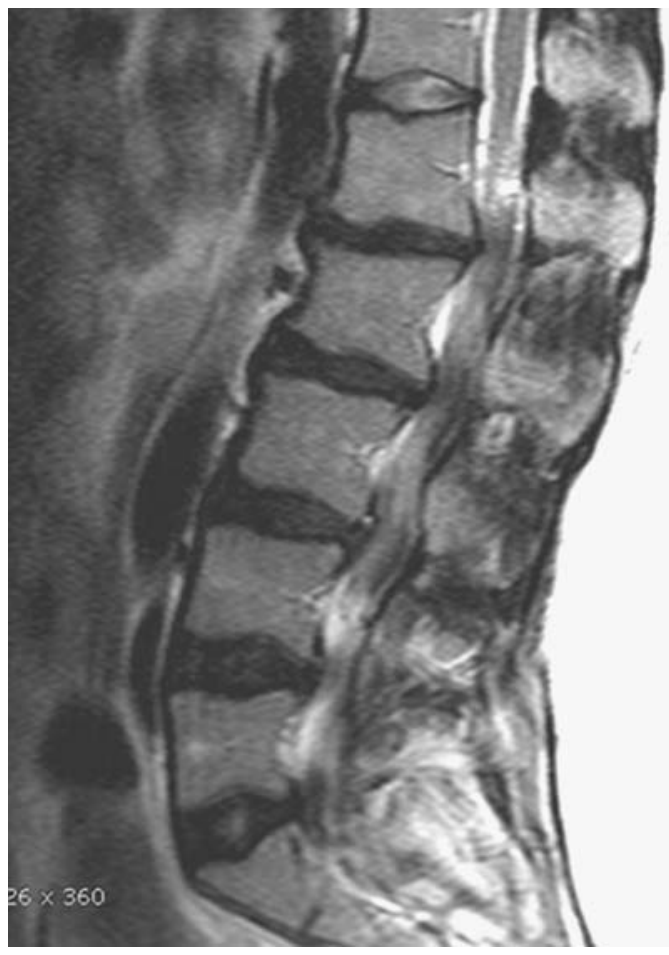

Fig. 3

T2-weighted mid-sagittal MR imaging in a symptomatic patient showing severe dural sac compression at the L1 to L2 and L2 to L3 intervertebral disc levels. The shadow of the cerebrospinal fluid column is absent.

the level of the vertebral body. The most common level affected was L1/2. None of the patients had stenosis at the L5/S1 level.

The degree of constriction of the spinal canal needed to produce symptoms of stenosis is unclear. Consequently, the definition of stenosis of the spinal canal varies. ${ }^{6}$ Verbiest $^{7}$ defined two subgroups of absolute stenosis (anteroposterior diameter on myelography $\leq 10 \mathrm{~mm}$ ) and relative stenosis (anteroposterior diameter between $10 \mathrm{~mm}$ and $12 \mathrm{~mm}$ ). Sortland et $\mathrm{al}^{8}$ described myelographic techniques in flexion and extension. They defined an anteroposterior diameter of $10.5 \mathrm{~mm}$ in extension as the lower limit of normal. To date, there is no agreement on the exact clinical or radiological definition of stenosis of the lumbar canal. Both clinical and radiological assessment are necessary to establish the diagnosis. ${ }^{9}$ Imaging can be used to determine the level of stenosis and its predominant subtype (i.e. central, lateral recess, foraminal or combined). A combination of these factors is used to assess a patient with spinal stenosis.

In 1984 , Schönstrom et $\mathrm{al}^{10}$ reported a study of spinal stenosis in the normal population with measurement of the anteroposterior canal by CT and measurement of the dural sac by myelography in patients undergoing surgery for stenosis. They found no correlation between the transverse area of the bony canal in normal patients and in the patients with stenosis. However, a dural sac cross-sectional area of
$100 \mathrm{~mm}^{2}$ or less correlated with symptomatic spinal stenosis.

Our results showed that in achondroplasia the mean sagittal diameter of the bony canal at the mid-body and the mid-disc levels was no different between symptomatic and asymptomatic patients except at the mid-body level at L1. The mean sagittal thecal diameter was no different in symptomatic and asymptomatic patients at both the mid-body and the mid-disc levels at any level. The significance of the sagittal dimension of the bony canal at the L1 mid-body level with no significant difference in the interpedicular distance at that level in the symptomatic group showed the contribution of the short pedicles in the causation of symptoms in these patients

The cross-sectional area of the spinal canal differed significantly between symptomatic and asymptomatic patients at both the mid-body and mid-disc levels at the L1 and L3 levels and at the mid-disc level at L2. Although this dimension did not differ significantly at the lower spinal levels (L4 and L5), most patients had some compression of the cauda equina at the upper spinal levels.

Our study suggests that achondroplasia patients with a narrow bony canal at the higher spinal levels (L1 to L3) are more likely to develop symptoms of spinal stenosis. The degenerative disc changes which occur in the intrinsically narrow canal are probably the reason why narrowing occurs more at higher lumbar levels. However, the small sample size limits the usefulness of our study and we are unable to formulate strict criteria for the diagnosis, quantification and management of stenosis of the lumbar canal in achondroplasia.

We were also hampered by the lack of dynamic MR images in flexion and extension which may have allowed us to evaluate the effect of position and loading on the spine.

In patients with achondroplasia, there is a significant correlation between the cross-sectional area of the spinal canal at higher lumbar levels and the development of symptoms of stenosis. This can be used as a radiological parameter to differentiate between symptomatic and asymptomatic patients. Individuals with a narrower spinal canal are at greater risk of developing the symptoms of spinal stenosis. Our study needs to be followed up by other larger studies so that guidelines may be formulated for the diagnosis, quantification and treatment of lumbar spinal stenosis in achondroplasia.

No benefits in any form have been received or will be received from a commercial party related directly or indirectly to the subject of this article.

\section{References}

1. Ruiz-Garcia M, Tovar-Baudin A, Del Castillo-Ruiz V, et al. Early detection of neurological manifestations in achondroplasia. Childs Nerve Syst 1997;13:208-13.

2. Yamada H, Nakamura S, Tajima M, Kageyama N. Neurological manifestations of pediatric achondroplasia. J Neurosurg 1981;54:49-57.

3. Yukawa Y, Lenke LG, Tenhula J, et al. A comprehensive study of patients with surgically treated lumbar spinal stenosis with neurogenic claudication. J Bone Joint Surg $[$ Am] 2002;84:1954-9.

4. Postacchini F. Surgical management of lumbar spinal stenosis. Spine 1999;24: 1043-7. 
5. Rollins N, Booth T, Shapiro K. The use of gated cine phase contrast and MR venography in achondroplasia. Childs Nerve Syst 2000;16:569-75.

6. Jånsson B, Annertz M, Sjoberg C, Strömquist B. A prospective and consecutive study of surgically treated lumbar spinal stenosis: Part 1. clinical features related to radiographic findings. Spine 1997;22:2932-7.

7. Verbiest H. Further experiences on the pathological influence of a developmental narrowness of the bony lumbar vertebral canal. J Bone Joint Surg [Br] 1955;37-B:576-83.
8. Sortland $\mathbf{O}$, Magnanes B, Hauge T. Functional myelography with metrizamide in the diagnosis of lumbar spinal stenosis. Acta Radiol Supp/ 1977;355:42-54.

9. Amundsen T, Weber $\mathbf{H}$, Lilleas F, et al. Lumbar spinal stenosis: clinical and radiologic features. Spine 1995;20:1178-86.

10. Schönstrom N, Boldender NF, Spengler DM, Hansson TH. Pressure changes within the causa equina following constriction of the dural sac: an in vitro study. Spine 1984;9:604-7 